\title{
Cardio-Pulmonary Exercise Testing Prior to Major Surgery
}

\author{
Michael R. Gooseman, MD, and Alessandro Brunelli, MD
}

Department of Thoracic Surgery, St. James University Hospital, Leeds Teaching Hospitals NHS Trust, Leeds, UK

The current issue of the Annals of Surgical Oncology publishes the excellent systematic review and meta-analysis of Sivakumar et al. ${ }^{1}$ which contributes to an increasing body of evidence supporting the role of cardio-pulmonary exercise testing (CPET) in the pre-operative assessment of patients undergoing major surgery. This work has demonstrated the value of incorporating CPET into the work-up for patients being submitted to oesphagectomy. The production of guidance relating to acceptable parameters deeming a patient safe to proceed with this operation may benefit from the growing experience in other aspects of chest surgery.

CPET is now accepted as the gold standard in functional assessment and risk stratification for patients being considered for major lung resection surgery. As Sivakumar et al. point out, the role of CPET in thoracic surgery has been studied for several decades. In the 1980s and 1990s there was a number of small studies that showed $\mathrm{VO}_{2} \max$ was inversely associated with mortality after lung resection. Bolliger et al. demonstrated that the percentage of predicted $\mathrm{VO}_{2}$ max was inversely associated with developing complications. There has also been extensive work comparing CPET with low technology testing such as stair climbing and shuttle walk testing. Despite the increasing data supporting CPET, it was initially limited in its utilisation. A 2009 European Respiratory Society/European Society of Thoracic Surgeons web-based survey showed that CPET technology was available in $75 \%$ of hospitals but only $10-30 \%$ of patients had CPET prior to lung

(C) Society of Surgical Oncology 2020

First Received: 8 May 2020;

Published Online: 23 May 2020

A. Brunelli, MD

e-mail: alex.brunelli@nhs.net resection. The problems facing CPET prior to oesphagectomy are therefore similar to what was faced in lung resection surgery.

The role of CPET prior to lung resection surgery became clearly defined in the American College of Chest Physicians (ACCP) guidance. Broadly speaking, patients are considered for CPET when they either have poor lung function, perform poorly on low technology exercise testing such as stair climbing, or have a significant cardiac risk assessment. Brunelli et al. ${ }^{2}$ contributed work that helped in defining thresholds for work regarding risk stratification. This group showed that patients with a $\mathrm{VO}_{2} \max >20 \mathrm{ml} /$ $\mathrm{kg} / \mathrm{min}$ undergoing lung resection had no mortality while the mortality rate was $13 \%$ in patients with a $\mathrm{VO}_{2} \max <$ $12 \mathrm{ml} / \mathrm{kg} / \mathrm{min}$. The guidance recommends that a patient with $\mathrm{VO}_{2} \max >20 \mathrm{ml} / \mathrm{kg} / \mathrm{min}$ can safely proceed with any form of surgical lung resection. It might be sensibly argued that the same can be considered for oesphagectomy patients.

Licker et al. demonstrated that patients undergoing lung resection with a $\mathrm{VO}_{2} \max <10 \mathrm{ml} / \mathrm{kg} / \mathrm{min}$ had a morbidity of $65 \%$. This work and others informed the current ACCP guidance that a $\mathrm{VO}_{2} \max <10 \mathrm{ml} / \mathrm{kg} / \mathrm{min}$ is generally viewed as a contraindication to lung resection surgery. A $\mathrm{VO}_{2} \max$ of between 10 and $15 \mathrm{ml} / \mathrm{kg} / \mathrm{min}$ is associated with increased risk of post-operative mortality. A metaanalysis and the studies included confirmed that this range was to be regarded as a high-risk range for lung resection. It is these patients that will often require further consideration in respect of suitability for surgery and management through the peri-operative process.

In the context of oesophagectomy, it is appreciated that this may be considered a more involved operation than a standard lung resection. However, one comparable operation, at least in the context of morbidity and mortality, is pneumonectomy. The European Society of Thoracic Surgeons (ESTS) database reported that pneumonectomy carries an in-hospital mortality of $6 \%$. Clinical guidelines 
do not have separate recommendations as to whether patients should proceed with this operation. However, given the mortality associated with the operation, it seems sensible to refer all pneumonectomy candidates to CPET. This principle could be reasonably applied to oesphagectomy patients.

The results of a CPET should not, of course, be used to either accept or reject patients for a surgical procedure. The aim of the physiological assessment is to allow for identification of those who can be accepted for surgery but present a high risk of peri-operative morbidity and mortality. It can be easily argued that operations like oesophagectomy and pneumonectomy immediately place patients at 'high risk'. However, this must be placed in the context that the operation in both cases may be the most effective treatment currently available. In practice, then, there always needs to be a balanced assessment between the need for obtaining the best treatment, patient risk and patient expectations.

High-risk surgical meetings are being adopted to help manage these patients. With the results available from preoperative investigations, including CPET, there can be benefit gained from review between the members of the wider peri-operative team including surgeon, anaesthetist and intensivist. The outcome can be personalised care pathways for the highest risk patients to streamline perioperative care with the aim of preventing complications before they arise. One of the other outcomes of these meetings can be the aim of optimising patients pre-operatively. Certainly, there may be benefits from smoking cessation programmes and pulmonary rehabilitation.

Major surgery will often carry significant risk of perioperative morbidity and mortality. This places particular emphasis on a thorough pre-operative evaluation and physiological evaluation. The work of Sivakumar et al. adds to the increasing evidence that CPET must be considered prior to major surgery to improve patient safety and outcomes.

DISCLOSURES The authors declare no conflicts of interest.

\section{REFERENCES}

1. Sivakumar J, Sivakumar H, Read M, Sinclair R, Snowden C, Hii $\mathrm{M}$. The role of cardiopulmonary exercise testing as a risk assessment tool in patients undergoing oesophagectomy: a systematic review and meta-analsysis. Anna Surg Oncol.

2. Brunelli A, Belardinelli R, Refai M, Salati M, Socci L, Pompili C, et al. Peak oxygen consumption during cardiopulmonary exercise test improves risk stratification in candidates to major lung resection. Chest. 2009; 135(5):1260-7.

Publisher's Note Springer Nature remains neutral with regard to jurisdictional claims in published maps and institutional affiliations. 